\title{
Image Findings and Common Musculoskeletal Disorders for Pediatrics in the Saudi Arabian Society
}

\author{
B. Z. Shakhreet \\ Department of Diagnostic Radiology, Faculty of Applied Medical Sciences, King Abdulaziz University, Jeddah, \\ Kingdom of Saudi Arabia \\ Email: bshakhreet@yahoo.com, bzshakhreet@kau.edu.sa
}

Received 12 March 2016; accepted 16 April 2016; published 19 April 2016

Copyright (C) 2016 by author and Scientific Research Publishing Inc.

This work is licensed under the Creative Commons Attribution International License (CC BY). http://creativecommons.org/licenses/by/4.0/

Open Access

\begin{abstract}
Aim: To detect the most common musculoskeletal disorders and to explore the common injury and indication of different musculoskeletal systems, and then to obtain the causes of disorders by observing clinical indications via images. Methodology: The subjects in this project consisted of both genders-males and females and were investigated randomly by collecting data from the PACS unit that exists in the radiology department at KAUH. The participants had a mean age of 7.4 \pm 0.3 years, a mean height of $118.2 \pm 2.5 \mathrm{~cm}$, a mean mass of $27.8 \pm 1.5 \mathrm{~kg}$, and a mean BMI of $19.6 \pm$ $0.8 \mathrm{~kg} / \mathrm{m}^{2}$. The author settled the study plan by building a special, appropriate questionnaire to be handed out to the patients and covered mainly the prospective studies of randomly selected patients who came to the emergency unit and the outpatient units from other departments at KAUH in Jeddah. Results: The ratio of injuries in male children (77\%) against females $(23 \%)$ is $3: 1$, and this ratio is considered significant between age groups for males when compared with females. The injuries existed most in the age groups of 4 to 6 years old and 8 to 10 years old with approximately the same percentage of the total subjects as about $21 \%$ for each age group. Trauma injury was significant (81\%) compared to other types of causes in all age groups. There was a significant relationship between the causes of injuries and the age groups in pediatrics $(p<0.05, \mathrm{df}=10)$ as well as the highest prevalence and significant relationship $\left(p=5.33 \times 10^{-6}\right)$ between the BMI and all causes of injuries in all pediatric age groups. The most occurred injuries were in the upper limb regions $(48.6 \%)$ and the other lower limb regions $(34.3 \%)$ in all age groups. There was no significant relationship $(p>0.05)$ between the site of injuries and age groups. The significant site that resulted from trauma was the knee $(100 \%)$, out of the total traumas' subject. There was a highly significant relationship between the site of injuries and its causes $(p=0.006$, i.e. $p<0.05)$ that were expected. Conclusion: More awareness and understanding in the society are needed and we should concentrate on increasing the knowledge of such by providing them with more learning courses.
\end{abstract}


Keywords

BMI, Pediatric Injuries, Musculoskeletal, Age Groups, Image Findings, Trauma, Sports, Accidents

\section{Introduction}

The household accidents and injuries involving children are a common and extremely regrettable phenomenon. Some believe that accidents and injuries are inevitable and cannot be avoided, but the information and facts confirm that this belief is a mistake. It is found that the lack of attention and caution is the main cause of these accidents. The proper attention and necessary precautions can help us avoid the risk of such accidents.

Children and their instincts are naturally inclined toward continuous discovery, so you see them trying to touch and examine things. They are also continuing to evolve and grow, and often surprise others that they have the ability to perform many movements, such as extending hands, volatility on the one hand to the other, keeping things, jumping, and other movements, in which children do not realize the dangers left behind. We, as parents and educators, should keep and preserve our children, and ensure a safe environment for them, taking into account the continuity of change and evolution in their needs and potentials.

Most of the pediatric injuries occur at home, and in general, children get injured when they try to practice new skills. The nature of a child leads a certain role where the active and curious child is more prone to accidents.

The lack of attention and carelessness of parents, the poor organization of the house, a busy area with equipment and unnecessary tools, and the lack of methods of protection and prevention, all contributes to the occurrence home injuries.

The presence of children in troubled families, family problems and frequent bad psychological cases as psychological stress or depression and unemployment or drug abuse also increase domestic accidents. The incidence and severity of injury are also high in some child and youth sports [1].

Many investigators and researchers had done different studies for pediatric musculoskeletal injuries. The relative frequency of sports-related injuries compared with all musculoskeletal injuries in patients 5 to 21 years of age presenting to the emergency unit (ER) was determined and then the evaluation of the sports-specific and anatomic site-specific nature of these injuries was performed [2].

Musculoskeletal injuries were more common in male patients (62\%) than in female patients. The mean age of the patients was 12.2 years. The most common injury types were sprains, contusions, and fractures $(34 \%, 30 \%$, and $25 \%$, respectively). Female patients experienced a greater percentage of sprains (44\% vs $36 \%$ ) and contusions (37\% vs $33 \%$ ) and fewer fractures (22\% vs $31 \%$ ) than male patients did. Sports injuries accounted for $41 \%$ of all musculoskeletal injuries and were responsible for $8 \%$ of all ER visits. Head, forearm and wrist injuries were most commonly seen in biking, hand injuries in football and basketball, knee injuries in soccer, and ankle and foot injuries in basketball.

Currently, Pediatric trauma remains a major cause of morbidity and mortality of children in the United States (U.S.) and requires exorbitant costs. The femur fracture was the most frequent injury among this patient group (21.7\% of orthopedic trauma), followed by tibia and/or fibula fracture (21.5\%), humorous fracture (17.0\%), radius and/or ulna fracture (14.8\%), and vertebral fracture (5.2\%). While the majority of pediatric orthopedic trauma was treated at non-children hospitals (70.4\%), patients with certain diagnoses such as femur, humorous, vertebral, pelvic or hand/finger fracture, or a back sprain/strain were directed to children's hospitals more frequently compared with the total number of pediatric orthopedic trauma patients [3].

Children who sustained a femoral shaft fracture in the 6 to 10-year age group were significantly more likely to receive internal fixation versus casting or traction if they were treated at a children's hospital. Understanding the patterns in which traumatic injuries occur in children is paramount to establishing effective injury prevention, as well as adapting treatment to optimize outcomes.

A number of 135 children and adolescents (68 girls, 67 boys) had been evaluated with a mean age of 12.3 years (range: 3 - 18 years old). The study population was Hispanic (51\%), non-Hispanic white (26\%), non-Hispanic black (13\%), and others (10\%). The majority of subjects (61\%) complained of at least one joint hurting more than once per month. Back pain was the most common complaint (39\%), followed by foot (26\%) and knee (24\%) pain. After adjustment for age, pain in the knees and hips were associated with increased weight 
and/or body mass index (BMI). Musculoskeletal pain was common in the knee and hip joints and was positively associated with extra bodyweight [4].

As for physical activity, participation is directly related to improvements in physical fitness, skeletal health and metabolic conditions. Higher levels of physical activity are encouraged, and exercise is commonly prescribed in the treatment and management of childhood obesity. It has been confirmed that overweight children typically display a slower, more tentative walking pattern with increased forces to the hip, knee and ankle during their "normal" gait. This work, combined with anthropometric data indicating a higher prevalence of musculoskeletal malalignment in overweight children, suggests that such individuals are poorly equipped to undertake certain forms of physical activity. Concomitant increases in obesity and decreases in physical activity levels strongly support the need to better understand the musculoskeletal factors associated with the performance of motor tasks by overweight and obese children [5].

In some pediatric ankle and wrist injuries, the clinical picture is suggestive of a fracture despite negative standard radiographs. So, the effectiveness of high-resolution ultrasound (US) in differentiating radiographically occult fractures from sprains had been determined and studied. Ultrasound is effective in detecting radiographically silent fractures of the pediatric ankle and wrist. Ultrasound may be used as an adjunct to radiography in clinically suspicious but radiographically negative ankle and wrist injuries. In ankle injury patients, the US did not reveal fractures in $63 \%$ of total ankle injury subjects while in $37 \%$ of them, small fractures were detected. It was noted that all patients with negative US studies had negative follow-up x-rays [6].

Although rarely mortal, falls from height carry a significant morbidity and are costly to the health care system. To decrease the occurrence of injuries caused by falls, strategies should include awareness campaigns, parents' education about the mechanisms of falls, increased parenteral supervision during play-activities, and legislative measures to ensure the safety of windows and balconies before the onset of summer [7].

Because the number of overuse-injuries is on the rise, it is important for the physician to be aware of the clinical manifestations of such injuries, to prescribe current recommended treatments, and to educate patients on proper athletic conditioning [8].

Sports activities are considered one of the causes of pediatric injury. Therefore, a survey of the injury rate for children in community sports was carried out. For all sports, there were more game than practice injuries; this difference was significant except for softball. In addition, there were no significant gender differences in soccer for injury rates during total events [9].

Traffic accidents are considered one of the most frequent factors and causes of injury. To study the impact of these injuries on society, of whether it would lead to natural and humanitarian disasters in the world, pedestrian versus motor vehicle accidents are associated with substantial morbidity and mortality. The demographics and injury profiles of pedestrian versus motor vehicle accidents in a large trauma system was examined. Patients were grouped by age: pediatric (less than 15 years), adult (15 to 65 years), and elderly (older than 65 years). The pediatric group represented $38.1 \%$ of the study population, adults $53.9 \%$, and the elderly $8.0 \%$ [10].

The largest database reported on the motor vehicle versus pedestrian accidents was contributed and it was found that these accidents are common in a large urban trauma system. Hospital stay, Injury Severity Score, Revised Trauma Score, Glasgow Coma Scale, and the mortality rate worsen with age. The high mortality rate among the elderly indicates the need for more aggressive and effective prevention efforts.

Other work aimed to determine the incidence of injuries of children participating in sports and to present advice on injury prevention. The highest incidence of sports injury was in the foot-ankle region, and the lowest incidence was in the hip-femur region. The incidences of injuries to the neck, shoulder, elbow, hand, wrist, superior dorsal region, waist, hip-femur region, knee, and foot-ankle regions weren't statistically significant. In the previous study, the causes of injuries were examined to propose preventive measures to minimize their occurrence and severity [11].

The recreational activities are important for a normal, healthy development of natural children. Participation in sports activities, games and entertainment increasingly grows each year, for children and adolescents. Many of these injuries are not serious and heal uneventfully; some of which can lead to permanent weaknesses. Minor injuries can also cause anxiety and pain for both children and parents, taking such costs in terms of time and money, and may lead to functional limitations [12].

Musculoskeletal disorders are considered one of the most general causes of disability for people all over the world. In adults, musculoskeletal pain is a common reason for care, especially in primary health care facilities, where it is usually assessed and managed. Therefore, a better understanding of these conditions in children and 
adolescents is important for the development of effective prevention strategies, and it provides a good knowledge of the development of chronic pain in adulthood and its origin phase [13].

Our study was conducted in order to detect the most common musculoskeletal disorder, which was found to be the fracture $(54.3 \%)$ in pediatric. This study also explored the common injury and indication of different musculoskeletal systems. It also obtained the causes of disorders by observing clinical indications via images and looked for major causes of common indications. It was found that the highest percentage (81\%) is related to trauma in the pediatric category.

We intend to extend our study in the future to further educate our community in preventing and limiting personal injury, especially in younger age groups. Reducing the common musculoskeletal indications by determining its causes and eliminating or reducing them will have economic consequences as they reduce the number of patients visiting the hospitals, in addition to decreasing the medical imaging services which is overcrowded with patients from outpatient clinics and emergency.

\section{Materials and Methods}

King Abdulaziz University Hospital (KAUH) issued an ethical approval through ethics commission to start and perform this survey study as well as collect the required data.

The researcher commenced the research by building a special, appropriate questionnaire to be given to the patients. He then covered mainly the prospective studies of randomly selected patients who came to the emergency unit and the outpatient units from other departments at KAUH in Jeddah.

The subjects in this project consist of both genders; males and females that were selected randomly by collecting data from the PACS unit that exists in the radiology department in KAUH. The participants had a mean age of $7.4 \pm 0.3$ years, a mean height of $118.2 \pm 2.5 \mathrm{~cm}$, a mean mass of $27.8 \pm 1.5 \mathrm{~kg}$, and a mean BMI of $19.6 \pm$ $0.8 \mathrm{~kg} / \mathrm{m}^{2}$.

The BMI was calculated by measuring the height in centimeters divided by body weight in kilograms (using a suitable meter machine and balance that was available at the department). After that, the calculations were categorized into underweight (under $18.5 \mathrm{~kg} / \mathrm{m}^{2}$ ), normal weight (18.5 to $\left.24.9 \mathrm{~kg} / \mathrm{m}^{2}\right)$, overweight (25 to $29.9 \mathrm{~kg} / \mathrm{m}^{2}$ ) and obese (over $30 \mathrm{~kg} / \mathrm{m}^{2}$ ) [14].

The questionnaire had two parts, the first part aimed to collect demographic data, while the second one was used to determine the incidences and regions (knee "K", upper limb regions "UP", other lower limb regions "LO", spine "SP" and pelvis "PL") of different types of injuries and disorders in pediatric age group. After the data collection, the author used an SPSS 15.0 program for statistical analyses purposes. Data are presented using descriptive statistics and were analyzed using the chi-squared test.

Normal radiographic images for different parts and organs were retrieved from the PACS, followed by printing the medical report and relying on both of them to fill our data sheets and questionnaire with the required information as illustrated in Figure 1. The figure shows some of the normal X-ray image findings for different regions in the human body such as; (a) knee for pediatric normal image, (b) anterior-posterior (AP) position of the right shoulder; usage as a reference for other injured regions for comparison and completing our study.

Later on, a health education program will be designed and readied for implementation in the future, to indi-

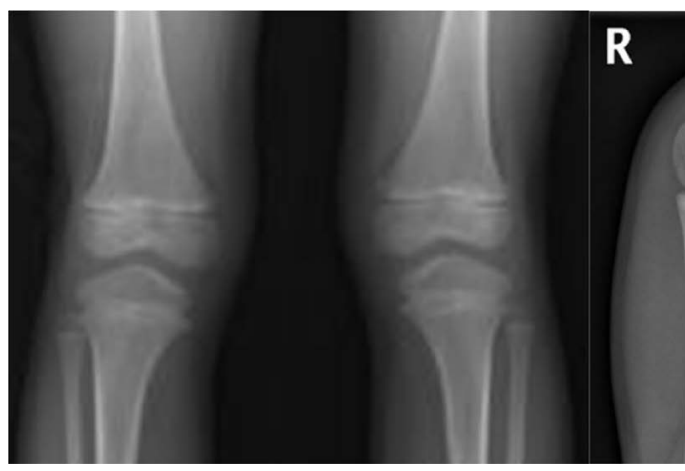

(a)

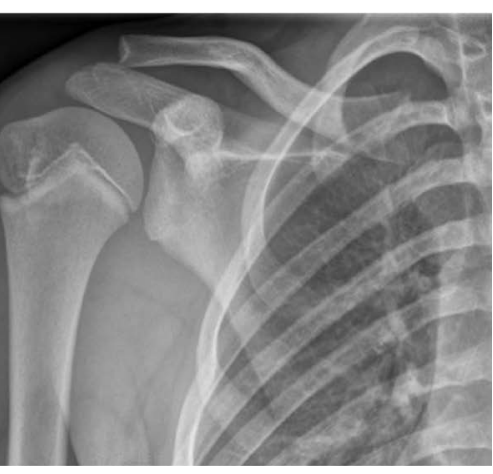

(b)

Figure 1. Normal X-ray image findings for different regions. 
cate the significant factors and best practices to prevent those injuries from happening, especially in focused groups of elderly people and children.

\section{Results}

The results of the current study showed that the rate of injuries in male children (77\%) against females (23\%) is 3:1, as this ratio is considered significant between the age groups for males, when compared with females.

The studied subjects were divided into seven age groups ranged from a newborn's age up to 14 years old and each group consisted of 2 years' period age. It was found that the most common injuries existed in the age groups of 4 - 6 years old and 8 - 10 years old, with approximately the same percentage of the total subjects as about 21\% for each age groups of them as it is illustrated in Figure 2. It was seen from Figure 1 that there were no cases or injuries in the newborn and infant age groups.

The results of the cause of injuries-occurrence are presented in Figure 3. It is seen that the trauma injury was significant (81\%) compared to the other types of causes in all age groups. The 4 - 6 years old age group showed the highest incidence of trauma injury (21\%), followed by the 6 - 8 years old age group (16\%) as well as the 8 -

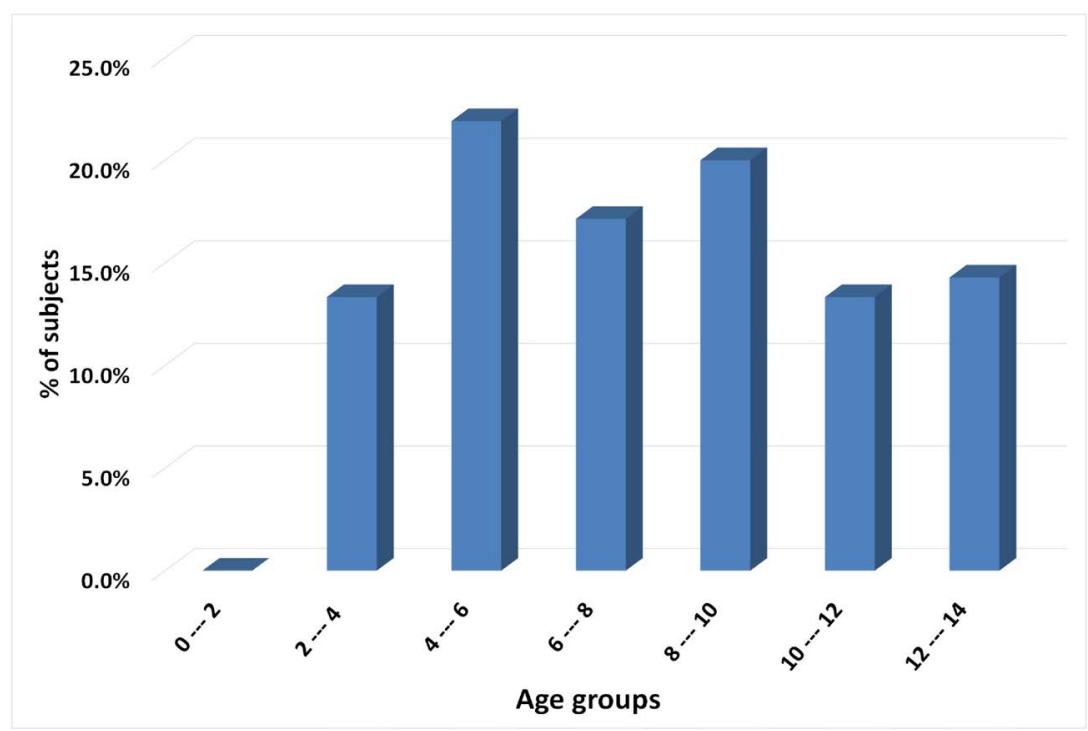

Figure 2. The percentage of subjects in relation to the pediatrics age group.

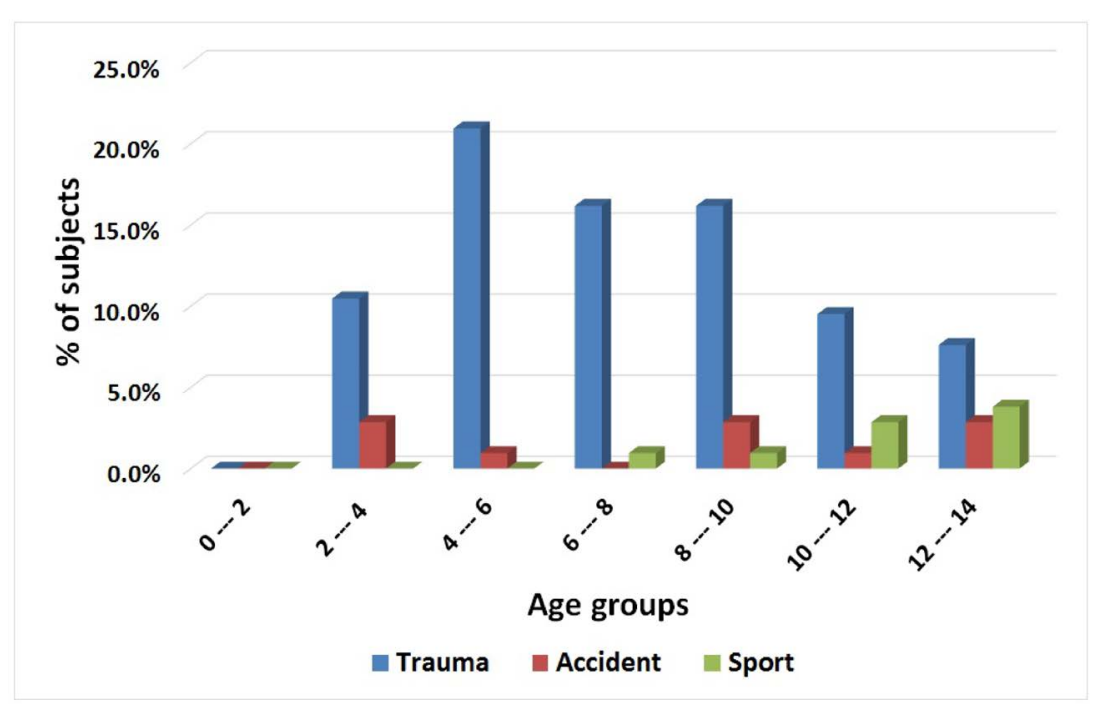

Figure 3. The percentage of causes of injuries in relation to the pediatrics age groups. 
10 years old age group. From our data in Figure 3, there is a significant relationship between the causes of injuries and the age groups in pediatrics $(\mathrm{p}<0.05, \mathrm{df}=10)$ where the highest significant relationship was for the age group of 12 - 14 years old.

Figure 4 displays the relationship between age groups and body mass index (BMI), as it is one of the objectives of this project. It is clear in the histograms in Figure 3 that most of the pediatric subjects fall in the underweight region, except for the age groups of 8 - 10 years and $10-12$ years old that most of the subjects fall in the normal weight region. Data shows the highest prevalence and significant relationship $\left(p=5.33 \times 10^{-6}\right)$ between the BMI and all causes of injuries in all pediatric age groups. The highest significant relationship $(\mathrm{p}=0.006$, i.e. $\mathrm{p}<0.05$ ) was for the $10-12$ years old's age group.

The relationship between age groups and site of injuries is shown in Figure 5. It can be seen from the figure that the most frequent injuries were in the upper limb regions (48.6\%) and the other lower limb regions (34.3\%) in all age groups. Also, the age group of $4-6$ years old had the upper limb regions injuries representing the highest ratio (10.5\%) of the total subjects' injuries as well as that of 12 - 14 years old. Injury incidences in the knee for pediatrics, in our study, are almost non-existent in all age groups with a minor percentage in two age groups (4 - 6 and 8 - 10 years old). From our data, it is noted there was no significant relationship ( $p>0.05$ ) between the site of injuries and age groups.

Generally, all causes of injuries have different incidence sites region in the human body. Figure 6 shows the relationship between the causes of injury and its sites. It is shown from the Figure that when trauma exists, the upper limb regions have the highest ratio of the total number of subjects ( $40 \%)$ followed by the other lower limb region (28.6\%). Also, trauma caused the most injuries in all sites in pediatrics, in this study.

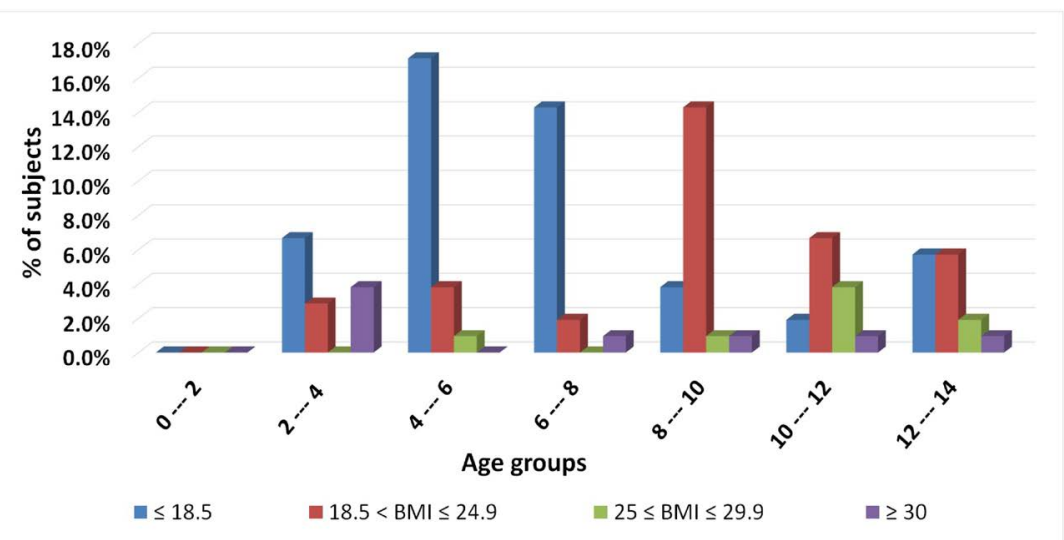

Figure 4. Illustrated BMI groups versus the pediatrics age groups.

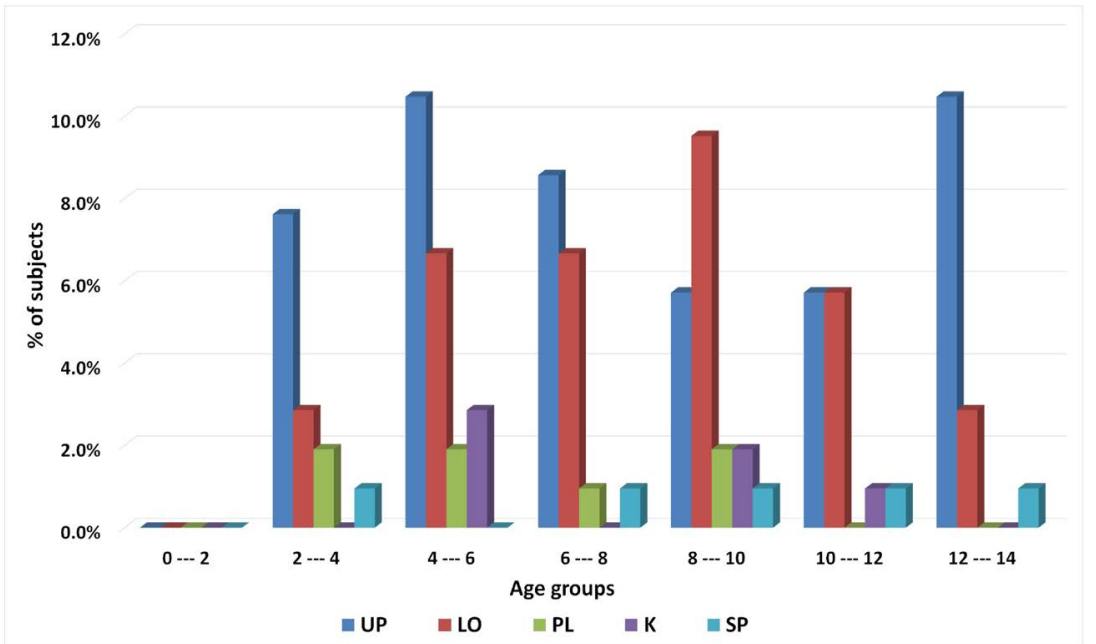

Figure 5. Site of injuries and its relationship with the age groups for pediatrics. 
If we compare each cause of injury with its sites that are affected with that cause, we can see that out of the total traumas' subject, the significant site that resulted from trauma was the knee $(100 \%)$. This is followed by the other lower limb regions (83.3\%) and the upper limb region (82.4\%), as shown in Figure 7. On the other hand, for the motor vehicle accident, the highest injured site was the spine (60\%) followed by pelvic injuries (28.6\%), other lower limb region (11.1\%) and upper limb region (3.9\%), with no cases injured in the knee site. In addition, all pediatric athletes in our study were only injured in the upper (13.7\%) and other lower limb regions (5.6\%). In fact, there was a highly significant relationship between the site of injuries and its causes ( $\mathrm{p}=$ 0.006 , i.e. $\mathrm{p}<0.05$ ) that were expected.

After the site of injuries and its causes were presented and determined in our study, the type of injuries (diagnosis) will be discussed and presented. There were different types of injuries that resulted in this survey study such as buckling, fracture, swelling, lesions, soft tissue abnormality, enlargement of the soft tissue of the palatine tonsils, narrowing of joint space and foreign body as tabulated in Table 1.

It is shown in the table that the most common musculoskeletal disorder is related to fracture (54.3\%) for all regions of injury and the highest significant number was in the upper limb region (37.1\%) followed by the other lower limb region (16.2\%). During this survey study, it was found that there was a normal case with a total per-

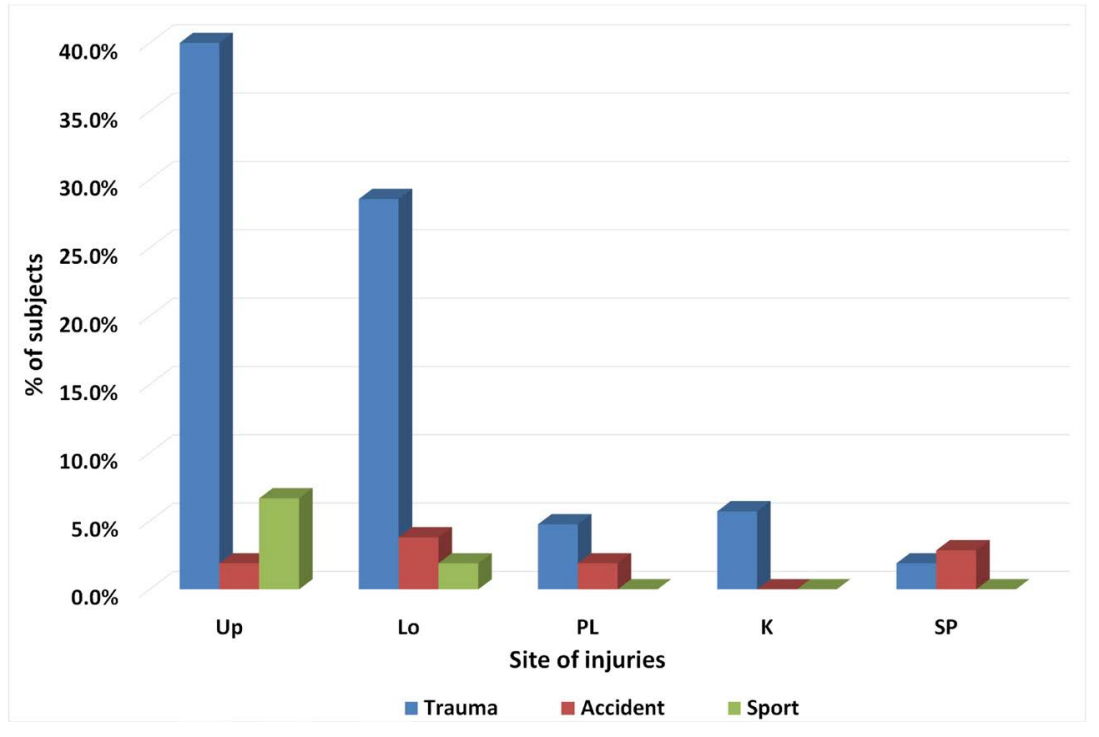

Figure 6. Site of injuries and its relationship with its causes in all pediatrics.

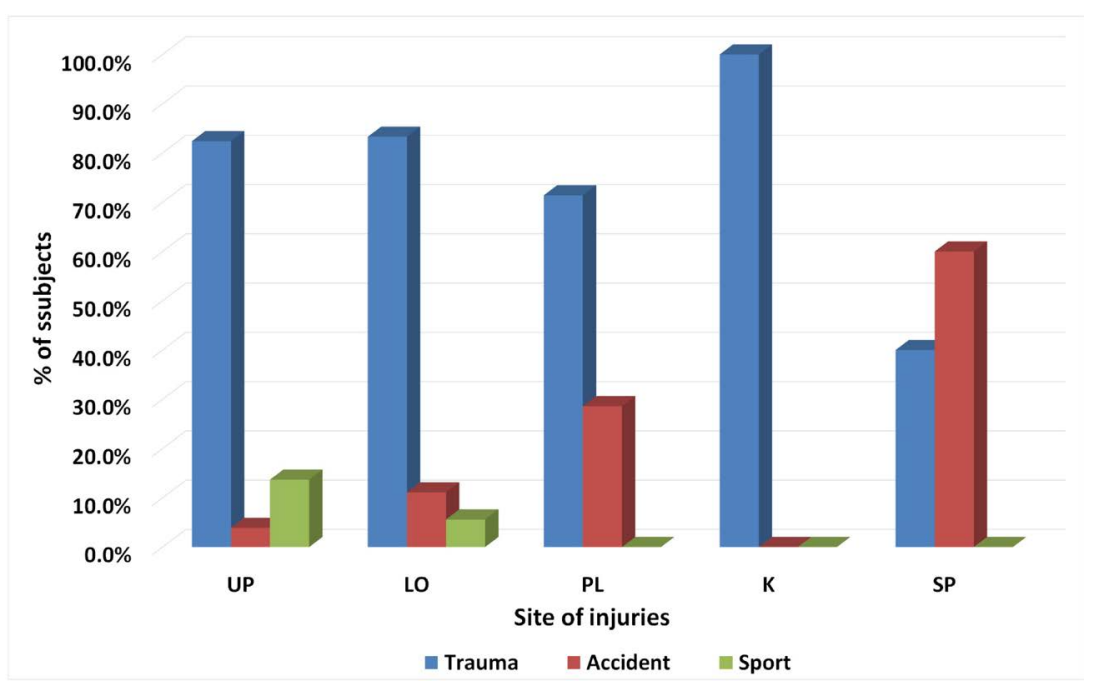

Figure 7. Ratio of injured sites for each cause of injury. 
Table 1. Relationship between type of injuries and its locations.

\begin{tabular}{|c|c|c|c|c|c|c|}
\hline \multirow{2}{*}{ Type of injury } & \multicolumn{5}{|c|}{ Site of injury } & \multirow{2}{*}{ Total } \\
\hline & UP & LO & PL & $\mathbf{K}$ & SP & \\
\hline Fracture & $37.1 \%$ & $16.2 \%$ & $0 \%$ & $1.9 \%$ & $0 \%$ & $54.3 \%$ \\
\hline Normal & $6.7 \%$ & $12.4 \%$ & $6.7 \%$ & $1 \%$ & $1.9 \%$ & $28.6 \%$ \\
\hline Swelling & $2.9 \%$ & $2.9 \%$ & $0 \%$ & $1.9 \%$ & $0 \%$ & $7.6 \%$ \\
\hline Buckling & $0 \%$ & $1 \%$ & $0 \%$ & $1.9 \%$ & $0 \%$ & $2.9 \%$ \\
\hline Foreign body & $0 \%$ & $1.9 \%$ & $0 \%$ & $0 \%$ & $0 \%$ & $1.9 \%$ \\
\hline Lesions & $1 \%$ & $0 \%$ & $0 \%$ & $0 \%$ & $0 \%$ & $1 \%$ \\
\hline Soft tissue abnormality & $1 \%$ & $0 \%$ & $0 \%$ & $0 \%$ & $1 \%$ & $1 \%$ \\
\hline Enlargement of palatine tonsils & $0 \%$ & $0 \%$ & $0 \%$ & $0 \%$ & $1 \%$ & $1 \%$ \\
\hline Narrowing of joint space & $0 \%$ & $0 \%$ & $0 \%$ & $0 \%$ & $1 \%$ & $1 \%$ \\
\hline
\end{tabular}

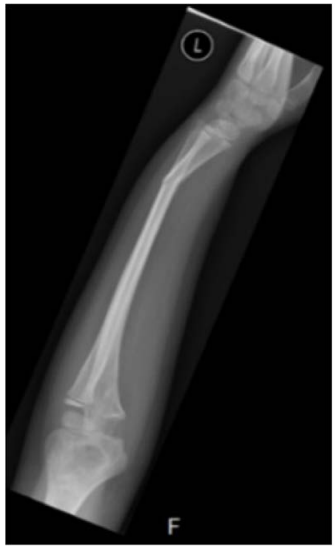

(a)

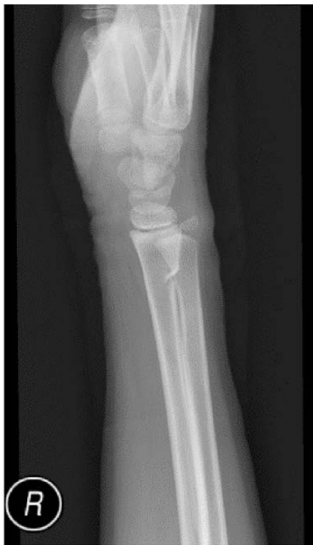

(b)

Figure 8. The pediatric upper limb fracture.

centage of $28.6 \%$ and were represented mostly the other lower limb regions (12.4\%) followed by the upper limb region (6.7\%) as well as the pelvic region.

These disorders (types of injuries) were examined and determined based on the normal X-ray image findings for different regions as explained in Figure 1. For example, in Figure 8(a) fracture (Figure 8(a)) and Buckling fracture (Figure 8(b)) in the upper limb region of the pediatric subject are illustrated.

\section{Discussion}

Children under 15 years of age in the Saudi society make up a large proportion of the population. It is known as well, that the large number of accidents and musculoskeletal injuries in this category are either because of traffic accidents or because of injuries that occur due to the exercise of free play, sports games or falls and other accidents. Therefore, it is very important to be accurate in the diagnosis and treatment of such injuries.

Many children are exposed to numerous accidents at home, whether due to negligence or bad use. The most domestic accidents result from falls due to causes such as scaling trees in their gardens, using the elevators incorrectly, climbing windows and walls of the balconies, the inappropriate use of games such as bicycles and swings, as well as slipping incidents. So, it is noted in this study that the majority of pediatric subjects were males (77\%) because of the active nature of males and frequent exposure to sports injuries at this age.

The significant musculoskeletal injuries in this study were related to the age group of pre-school age (4 - 6 years old) that was $21 \%$ of the total study subjects. Children at this age lack the motor skills to maneuver the knowledge and skills needed to differentiate the threat from safety. It is clear that the child's age is predictive of risk and the type of injury. Infants are more vulnerable to serious injuries due to suffocation, motor vehicle accidents, drowning, and burns. Children and preschoolers are more likely to die from drowning, car collisions, 
fires and burns, or suffocation. The school-age child is more likely to engage in risk-taking behaviors that lead to pedestrian injuries, bicycle injuries, drowning, and unintentional firearm injuries. Motor vehicle occupant injury continues to be the most significant mechanism of injury for this age group, as well as for the adolescent.

The child has the right to live a decent life. This comes only through respect for the child's individuality and so all aspects of his personality are developed by giving him the freedom to practice his favorite exercises and activities, as the child at this stage learns by playing; but he may be exposed to the problems or incidents during the performance of these games. So, parents should accompany their children, guide and supervise them to achieve the aims of the playing and exercises, which lets them learn without problems.

It was noticeable that the pathological indications of the musculoskeletal system had increased possibly due to several factors such as modern lifestyle, increase in car accident cases, modern sports and games and more hyperactivity in children due to video games' violence.

Unfortunately, with intense regret, despite all the alerts, warnings and instructions, the proportion of children who suffer from trauma, whether at home or abroad was a significant $81 \%$ of the current study sample. The age group of 4 - 6 years old had the significant proportion of $21 \%$ of the trauma injury. Generally, in the local community, it was found that trauma is a significant $53 \%$ for the age group of $4-10$ years old. It resulted in the fact that there is a significant relationship between the causes of injuries such as trauma, motor vehicle accident and sports, and the age groups in pediatrics $(\mathrm{p}<0.05, \mathrm{df}=10)$. That means, awareness and understanding should be there in our home and society to protect our children from risks.

Child-incident and injury problems are considered as a painfully tragic result, and this is due to the lack of accurate control of their daily behaviors by parents or school. A lack of awareness and evaluation of the consequences of the child's actions may affect him or hurt others.

There are many ways to prevent and avoid the consequences harm. The awareness of parents and their culture have played an important role in the prevention of accidents. As a proof of that, many of these incidents exist in underdeveloped and poor countries and environments, where there is a declining degree of culture, especially mothers.

In those countries' situations, we can recognize a lot of child negligence where parents allow them to move on their mood, without censorship, either inside or outside the home. We also observe children under the age of five who play in the alleys, lanes and between the cars without any concern from parents about the hazards.

Moreover, obesity and over weightness are increasing in the Kingdom of Saudi Arabia (KSA) with an overall obesity prevalence of $35.5 \%$ where the number of obese children is about three and a half million (12.2\%) children. Reduction in overweight and obesity are of considerable importance to public health [15]. There is a significant association between high BMI and sports injuries in most of the age groups. The prevalence of athletes and also the incidence of injuries is lower amongst the elderly [16].

In our study, it was found that the highest prevalence and significant relationship $\left(p=5.33 \times 10^{-6}\right)$ existed between the BMI and all causes of injuries in all pediatric age groups where the highest significant relationship $(\mathrm{p}=0.006$, i.e. $\mathrm{p}<0.05)$ was for the age group of $10-12$ years old.

It is clear that obesity in children play an important role in musculoskeletal injuries. Obesity is a result of malnutrition or an inappropriate nutrition behavior of children. It occurs because of the existence of an enormous number of calories found in some simple carbohydrates such as sweets, biscuits, muffins and cakes or because it contains high levels of fat gained from the fast food eaten outside or requested at home.

Although the upper limb region and other lower limb regions' injuries were significant for all age groups, there was no significant relationship $(p>0.05)$ between the age groups and site of injuries. That means injury can exist in any organ or region regardless of the age.

Generally, it is well known that the movements, exercises and sports are important and useful, especially for children, to help them get proper growth, build a healthy body and have strong bones. In fact, there was a highly significant relationship between the site of injuries and its causes $(p=0.006$, i.e. $\mathrm{p}<0.05)$ that were expected. Therefore, the knee, for example, has the highest and is the major region that is affected by trauma (100\%) regardless of how or where.

Knee injuries usually occur in sports that require jumping, which bends the knee, or when the knee is used for kicking the ball like soccer, gymnastics and running. Sports that involve sitting in a squatting position like weight lifting and body building also come in to play. In some communities that would normally sit in the status of bending the knee for long periods, such as sitting on the floor for long periods, it also displays infections in the tendons. When inflammation occurs, for the vast majority of patients, it is concentrated at the confluence of 
the tendon's bottom part of the greatness of the kneecap. Because in some cases, the child is still in the growth phase, this part of the kneecap may not be completely hardened and have turned into a harsh bone, as is the case in the adult. Therefore, it is susceptible to these infections.

The spine injuries are considered the most serious injuries that can expose the human as a result of an accident, especially cervical spine injuries at the top of the spine, due to complications arising from them, including quadriplegia or paraplegia injury, which causes health, psychological and social problems and harm. The region and sites most affected by motor vehicle accident were spine injuries (60\%) followed by pelvic injuries (28.6\%).

As a result, the positive cases that have fractures were the vast majority (54.3\%) for all children in this study where the most affected region was the upper limb region (37.1\%) while the negative cases (normal cases) were just $28.6 \%$.

\section{Conclusions}

Through the results of this study, it was found that there was a significant relationship between the BMI and causes of musculoskeletal injuries for all pediatric age groups. To avoid the increasing ratio of obesity in pediatrics, recommendations and more education must be provided to the parents and their children as well.

Knee injury often occurs among children and adolescents, especially males, because the majority of sports activities in schools rely on football, which is one of the reasons for the appearance of these injuries. The duty of parents, as well as a sports teacher is to pay attention to these symptoms, and to not force the child to practice the sport in case of symptoms associated with those injuries. They should also try to instill a culture of warm-up before the sport, for these children.

Parents also should consult a doctor in case of continuation of these symptoms, so that the necessary remedial action plan is taken. After the treatment of this problem, parents must choose the appropriate sport for their child, in order to ensure non-recurrence of the problem and avoid a chronic disease in the region. It should have alerted, that in some cases, there is a problem with the cartilage growth of the kneecap. This may cause the doctor to resort to using a medical splint for a short period, or to prevent a child from practicing violent sports and soccer practice for a few weeks or a few months in order to ensure the area heals completely.

In fact, through this survey study, it is found that the spine injuries are considered the most serious injuries that can expose the human as a result of a motor vehicle accident. This occurs especially in cervical spine injuries at the top of the spine, due to complications arising from them, including quadriplegia or paraplegia injury, which causes health, psychological and social problems and harms.

For traffic accidents and their relationship to injuries of the musculoskeletal system, KSA is doing awareness campaigns to warn about road accidents, because the mortality rate has reached great limits and has become a threat that needs confrontation.

To prevent pedestrian accidents, parents personally should personally send their children to school. This is especially if they are young, and the child should be placed on the exterior of the road. The child must walk in the direction opposite to the car's direction, walk on the sidewalk, avoid using any headset in his ear so that he can hear the vehicles' horn and the alerts of traffic cops, use the footbridge, and ask for help and assistance from the traffic cop to stop the traffic.

From another point, parents must send their children into the school campus using the areas specifically allocated for them. They may also be driving cautiously and not resort to the speed even in the case of delay and alert their children on how to wait for them when they are waiting outside of the school compound.

Children, at a young age, are unable to protect themselves from accidents, and they are very innocent. We must help them to make the innocence of their world safer and more secure.

The safety of the child and incidents of children are some of the things that have no limits or a certain age, where the child is independent of his family. Children in the early stages are trying to explore around them through their growth where curiosity has no boundaries with the lack of awareness of the danger.

Any simple neglect from the father or mother leads to a serious musculoskeletal injury or permanent disability.

Of course, trauma is one of the causes of musculoskeletal injuries as it is explained previously from the resulted data. Nevertheless, there are other main causes behind trauma such as the lack of awareness and lack of maternal experience for mothers in ways that protect their children from the household dangers and failure to follow security means to protect children. Additionally, the failure to take necessary measures to prevent children from falling or slipping inside homes also counts. 
Finally, children's behaviors cause them to fall into problems, and lead them to incidents that require more care. Therefore, we have to take into account the means and possibilities that the child has to deal with it and the availability of safety and security factors. The child may play with anything without realizing that it is a source of harm to him. It should be noted that the child must be observed and not left alone. The availability of safety and security in the atmosphere must be there, both inside the house or in kindergarten.

Based on all results, more awareness and understanding in the society is needed and we should concentrate on increasing the knowledge for parents by providing them with more learning courses. Therefore, it is important to expand this study in the future, to continue the education of our society in order to prevent personal injury and to reduce it for children, thus reducing the proportion of musculoskeletal injuries. This future work will have positive economic consequences of the task, because it will reduce the number of musculoskeletal injured children who visit hospitals. In addition, it will reduce the medical imaging services, which are often overcrowded with patients from outpatient clinics and emergency units.

\section{References}

[1] Caine, D., Caine, C. and Maffulli, N. (2006) Incidence and Distribution of Pediatric Sport-Related Injuries. Clinical Journal of Sports Medicine, 16, 500-513. http://dx.doi.org/10.1097/01.jsm.0000251181.36582.a0

[2] Dorothy, T.D., Jordan, M.D., Maria, R., et al. (2003) Patterns in Childhood Sports Injury. Pediatric Emergency Care, 19, 65-67. http://dx.doi.org/10.1097/00006565-200304000-00001

[3] Gregory, G.J., Mark, V.A., Michael, K.W., et al. (2005) The Most Frequent Traumatic Orthopedic Injuries from a National Pediatric Inpatient Population. Journal of Pediatric Orthopedics, 25, 39-44.

[4] Steven, D.S., Perrie, E.P., Gabriela, V., Sue, D. and Jeffrey, B.S. (2008) Musculoskeletal Pain in Obese Children and Adolescents. Acta Paediatrica, 97, 489-493. http://dx.doi.org/10.1111/j.1651-2227.2008.00724.x

[5] Shultz, S.P., Anner, J. and Hills, A.P. (2009) Pediatric Obesity, Physical Activity and the Musculoskeletal System. Obesity Reviews, 10, 576-582. http://dx.doi.org/10.1111/j.1467-789X.2009.00587.x

[6] Simanovsky, N., Lamdan, R., Hiller, N. and Simanovsky, N. (2009) Sonographic Detection of Radiographically Occult Fractures in Pediatric Ankle and Wrist Injuries. Journal of Pediatric Orthopedics, 29, 142-145. http://dx.doi.org/10.1097/BPO.0b013e318198452e

[7] Lallier, M., Bouchard, S., St-Vil, D., Dupont, J. and Tucci, M. (1999) Falls from Heights among Children: A Retrospective Review. Journal of Pediatric Surgery, 34, 1060-1063. http://dx.doi.org/10.1016/S0022-3468(99)90564-X

[8] Jennifer, L. and Jennifer, W.J. (2004) Overuse Injuries in Pediatric Athletes. Current Opinion in Pediatrics, 16, 47-50. http://dx.doi.org/10.1097/00008480-200402000-00009

[9] Radelet, M.A., Lephart, S.M., Rubinstein, E.N. and Myers, J.B. (2002) Survey of the Injury Rate for Children in Community Sports. Pediatrics, 110, e28. http://dx.doi.org/10.1542/peds.110.3.e28

[10] Peng, R.Y. and Bongard, F.S. (1999) Pedestrian versus Motor Vehicle Accidents: An Analysis of 5000 Patients. Journal of the American College of Surgeons, 189, 343-348. http://dx.doi.org/10.1016/S1072-7515(99)00166-0

[11] Atay, E. (2014) Prevalence of Sports Injuries among Middle School Children and Suggestions for Their Prevention. Journal of Physical Therapy Science, 26, 1455-1457. http://dx.doi.org/10.1589/jpts.26.1455

[12] Purvis, J. and Burke, R. (2001) Recreational Injuries in Children: Incidence and Prevention. Journal of the American Academy of Orthopedic Surgeons, 9, 365-374. http://dx.doi.org/10.5435/00124635-200111000-00002

[13] Henschke, N, Harrison, C., McKay, D., et al. (2014) Musculoskeletal Conditions in Children and Adolescents Managed in Australian Primary Care. BMC Musculoskeletal Disorders, 15, 164. http://dx.doi.org/10.1186/1471-2474-15-164

[14] Aim for Healthy Weight. National heart, Lung and Blood Institute. US Department of Health and Human Services. http://www.nhlbi.nih.gov/health/educational/lose_wt/BMI/bmicalc.htm

[15] Al-Nozha, M.M., Al-Mazrou, Y.Y., Al-Maatouq, M.A., et al. (2005) Obesity in Saudi Arabia. Journal of Saudi Medicine, 26, 824-829.

[16] Nagadi, D., Shakhreet, B.Z., Jastaniah, S., Khashoggi, K. and Elkhedr, A. (2016) Common Musculoskeletal Injuries in Athletes and Its Relation with Body Mass Index (BMI) in Jeddah Society. British Journal of Medicine \& Medical Research, 13, 1-6. http://dx.doi.org/10.9734/BJMMR/2016/24500 